\title{
Enhancement of adaptive observer robustness applying sliding mode techniques
}

\author{
Denis Efimov ${ }^{\text {a }}$ Christopher Edwards ${ }^{\mathrm{b}}$ Ali Zolghadri ${ }^{\mathrm{c}}$ \\ ${ }^{a}$ Non-A team at Inria Lille, Parc Scientifique de la Haute Borne, 40 avenue Halley, 59650 Villeneuve d'Ascq, France (e-mail: \\ Denis.Efimov@inria.fr) \\ ${ }^{\mathrm{b}}$ College of Engineering Mathematics and Physical Sciences, University of Exeter, Exeter EX4 4QF, UK (e-mail: \\ C.Edwards@exeter.ac.uk) \\ ${ }^{\mathrm{c}}$ University of Bordeaux, IMS-lab, Automatic control group, 351 cours de la libération, 33405 Talence, France (e-mail: \\ Ali.Zolghadri@ims-bordeaux.fr)
}

\begin{abstract}
The problem studied in this paper is one of improving the performance of a class of adaptive observer in the presence of exogenous disturbances. The $H_{\infty}$ gains of both, a conventional and the newly proposed sliding-mode adaptive observer, are evaluated to assess the effect of disturbances on the estimation errors. It is shown that if the disturbance is "matched" in the plant equations, then including an additional sliding-mode feedback injection term, dependent on the plant output, improves the accuracy of observation.
\end{abstract}

\section{Introduction}

The problem of adaptive observer design for nonlinear systems is an active research topic that finds many applications in various engineering fields. Typically, the observer needs to generate estimates of the vector of unknown parameters and unmeasured state components under noisy environments (see for example [4,14]). Highgain observers with gain adaptation for time-varying or nonlinear systems have been studied in a number of recently published papers, see for instance $[5,15,1,10]$.

An important issue is the relative degree between the output signal and the vector of unknown parameters (i.e. the number of derivatives of the output required, before the direct dependence on the vector of unknown parameters is obtained). Observers designed in the case when the degree is one [12] and for the higher relative degree case $[13,17,20,9]$ have completely different structures, and the dimension of the observers in the latter case is much higher.

There exist a number of potential solutions aimed at improving the robustness in nonlinear systems by applying dynamic or static feedback. Some very promising solutions have been obtained in the area of sliding mode theory, since sliding mode feedback is able to fully compensate for matched disturbances granting the closed loop system finite-time stability [16]. Recently the sliding mode approach has been successfully applied to adaptive observer design in the case of relative degree one systems [18], but the extension of this theory for adaptive observer design with a high relative degree is complicated due to the fixed observer structure.

In this paper a method is presented for augmenting the adaptive observer from [20], using sliding mode feedback, to cope with matched uncertainties in the spirit of [18]. The resulting solution ensures that the level of observer robustness with respect to some matched disturbances is improved.

\section{Problem statement}

Consider the following uncertain nonlinear system:

$$
\dot{x}=A x+\phi(y, u)+G(y, u) \theta+B v, y=C x,
$$

where $x \in \mathbb{R}^{n}, y \in \mathbb{R}^{p}, u \in \mathbb{R}^{m}$ are the state, the output and the control respectively, $\theta \in \mathbb{R}^{q}$ is the vector of unknown parameters; $v \in \mathbb{R}^{s}$ is the vector of external disturbances and $v: \mathbb{R}_{+} \rightarrow \mathbb{R}^{s}$ is a (Lebesgue) measurable function of time; the matrices $A, B, C$ are known and are assumed to have appropriate dimensions (and the pair $(A, C)$ is detectable); the functions $\phi: \mathbb{R}^{p+m} \rightarrow \mathbb{R}^{n}$ and $G: \mathbb{R}^{p+m} \rightarrow \mathbb{R}^{n \times q}$ are also assumed to be known and 
ensure uniqueness and existence of solutions to system (1) at least locally.

The symbol $|x|$ denotes the Euclidean norm of a vector $x$ (for a matrix $A$ the symbol $|A|$ denotes the induced matrix norm), and for the (Lebesgue) measurable functions $v: \mathbb{R}_{+} \rightarrow \mathbb{R}^{s}$, the norm is defined as $\|v\|=\operatorname{ess~sup}_{t \geq 0}\{|v(t)|\}$. For a matrix function $A$ : $\mathbb{R}_{+} \rightarrow \mathbb{R}^{s \times q}$ we denote $\|A\|=\||A(t)|\|$. The identity matrix of dimension $n \times n$ is denoted as $I_{n}$ and the symbols $\lambda_{\min }(A), \lambda_{\max }(A)$ represent the minimal and maximal eigenvalues of a symmetric matrix $A \in \mathbb{R}^{n \times n}$.

In this work we will assume that certain signals in the system (1) are bounded:

Assumption $1\|v\|<+\infty,\|G(y, u)\|<+\infty$.

Although assumed bounded, the disturbance $v$ may have a large magnitude, and therefore special attenuation techniques have to be applied to ensure reliable estimates for the states in system (1).

The objective of this work is to design an adaptive observer for (1) under the conditions of Assumption 1. The observer has to provide estimates of the vectors $x$ and $\theta$ with an enhanced degree of robustness with respect to the external disturbance $v$. The proposed design procedure is completed in two steps. Firstly, an adaptive observer is designed and the $H_{\infty}$ gain between the disturbances and the output errors is calculated. Secondly, an additional sliding mode output injection is applied to further reduce the influence of the disturbance components which cannot be completely rejected by the first step.

An adaptive observer for the system (1) has been proposed in [20], and takes the form:

$$
\dot{z}=A z+\phi(y, u)+G(y, u) \hat{\theta}+L(y-C z)+\Omega \dot{\hat{\theta}},
$$

where

$$
\begin{gathered}
\dot{\Omega}=(A-L C) \Omega+G(y, u), \\
\dot{\hat{\theta}}=\gamma \Omega^{T} C^{T}(y-C z) .
\end{gathered}
$$

In (2)-(3), $z \in \mathbb{R}^{n}$ is the estimate of $x, \hat{\theta} \in \mathbb{R}^{q}$ is the estimate of $\theta$, and $\Omega \in \mathbb{R}^{n \times q}$ is an auxiliary/filter variable, that helps to overcome possible high relative degree obstructions in system (1). In (3) $\gamma>0$ is a design parameter, and $L$ is the observer gain that is chosen to ensure a Hurwitz property for the matrix $A-L C$. The analysis of the estimation abilities of the observer in (2), (3) is based on the errors $\delta=x-z+\Omega \tilde{\theta}$ and $\tilde{\theta}=\hat{\theta}-\theta$ whose dynamics can be shown to have the form:

$\dot{\delta}=(A-L C) \delta+B v$,

$\dot{\tilde{\theta}}=\gamma \Omega^{T} C^{T}(C \delta-C \Omega \tilde{\theta})$.
From equation (4) we conclude that $\delta(t) \underset{t \rightarrow+\infty}{\longrightarrow} 0$ for $v=0$ and the variable $\delta$ stays bounded for any bounded disturbance $v$. From (3) the Hurwitz property of the matrix $A-L C$ and Assumption 1 imply boundedness of the variable $\Omega$. If the signal $G(y, u)$ is persistently exciting $(\mathrm{PE})[3,19]$, then due to the filtering property of the variable $\Omega$, the variable $C \Omega$ is also $\mathrm{PE}$. Moreover, it is possible to show [9] that for any bounded signal $C \delta$, the variable $\tilde{\theta}$ has a bounded response, and if $C \delta(t) \underset{t \rightarrow+\infty}{\longrightarrow} 0$, then $\tilde{\theta}(t) \underset{t \rightarrow+\infty}{\longrightarrow} 0$ also. This "proof" is based on general stability arguments, and no strict Lyapunov function has been proposed (this drawback will be overcame later in the present work).

\section{Conventional adaptive observer}

First let us show that the system in (5) is input-to-state stable with respect to the input $C \delta$.

Lemma 1 [8] Let the variable $\Omega^{T} C^{T}$ be $P E$ and bounded, i.e. $0<\rho=\|C \Omega\|<+\infty$ and there exist constants $\vartheta>0$ and $\ell>0$ such that

$$
\int_{t}^{t+\ell} \Omega(\tau)^{T} C^{T} C \Omega(\tau) d \tau \geq \vartheta I_{q} \quad \forall t \geq 0 .
$$

Then

a) there exists a continuous symmetric matrix function $P: \mathbb{R}_{+} \rightarrow \mathbb{R}^{q \times q}$ such that $\rho^{-2} I_{q} \leq 2 \gamma P(t) \leq \alpha I_{q}$ for all $t \geq 0$, where the scalar $\alpha=\gamma \eta^{-1} e^{2 \eta \ell}$ and $\eta=$ $-0.5 \ell^{-1} \ln \left(1-\frac{\gamma \vartheta}{1+\gamma^{2} \ell^{2} \rho^{4}}\right)$;

b) for all $t \geq 0$

$$
\dot{P}(t)-\gamma P(t) \Omega(t)^{T} C^{T} C \Omega(t)-\gamma \Omega(t)^{T} C^{T} C \Omega(t) P(t)+I_{q}=0 ;
$$

c) for $S(t, \tilde{\theta})=\tilde{\theta}^{T} P(t) \tilde{\theta}$ we have for all $\tilde{\theta} \in \mathbb{R}^{q}, \delta \in \mathbb{R}^{n}$ and $t \geq 0$

$$
\dot{S} \leq-\gamma \alpha^{-1} S+0.5 \rho^{2} \alpha^{2}|C \delta|^{2}
$$

In addition, for all $\tilde{\theta}(0) \in \mathbb{R}^{q}$ and all $t \geq 0$ the following estimate is satisfied:

$$
|\tilde{\theta}(t)| \leq \rho \sqrt{\alpha}\left[e^{-0.5 \gamma \alpha^{-1} t}|\tilde{\theta}(0)|+\rho \alpha\|C \delta\|\right] .
$$

Remark 2 This lemma also provides an estimate on the fastest rate of decrease of the parametric estimation error $\tilde{\theta}(t)$. Specifically, the rate of decrease equals $0.5 \gamma \alpha^{-1}=$ $0.5 \eta e^{-2 \eta \ell}=-0.25 \ell^{-1} \ln \left(1-\frac{\gamma \vartheta}{1+\gamma^{2} \ell^{2} \rho^{4}}\right)\left(1-\frac{\gamma \vartheta}{1+\gamma^{2} \ell^{2} \rho^{4}}\right)=$ $g(\gamma)$. The mapping $g$ is a function of $\gamma$ dependent on 
parameters $\vartheta>0, \ell>0$ and $0<\rho=\|C \Omega\|<+\infty$ of the PE variable $\Omega^{T} C^{T}$. Computing the derivative of $g$ we obtain:

$$
\frac{\partial g}{\partial \gamma}=0.25 \ell^{-1} \vartheta \frac{1-\gamma^{2} \ell^{2} \rho^{4}}{\left(1+\gamma^{2} \ell^{2} \rho^{4}\right)^{2}}\left(1+\ln \left(1-\frac{\gamma \vartheta}{1+\gamma^{2} \ell^{2} \rho^{4}}\right)\right) .
$$

Since $\vartheta \leq \ell \rho^{2}$ from the definition of the PE property, then the equation $\frac{\partial g}{\partial \gamma}=0$ has just one solution

$$
\gamma_{o p t}=\ell^{-1} \rho^{-2}
$$

which gives the maximum rate of convergence $g\left(\gamma_{\text {opt }}\right)=$ $-\frac{1}{4 \ell} \ln \left(1-\frac{\vartheta}{2 \ell \rho^{2}}\right)\left(1-\frac{\vartheta}{2 \ell \rho^{2}}\right)$ of the parametric estimation error $\tilde{\theta}(t)$. Increasing the convergence rate implies a decrease in $\alpha$, and also decreases the value of the $H_{\infty}$ gain.

Note that in order to use these estimates we have to know the values of $\rho, \ell$ and $\vartheta$. The existence of such a $\rho$ follows from Assumption 1 for the Hurwitz matrix $A-L C$, but to compute it we have to know $\|x\|$, which is assumed to be unavailable. However, the values of $\rho, \ell$ and $\vartheta$ can all be evaluated numerically during experiments by computing the integral of $\Omega(t)^{T} C^{T} C \Omega(t)$ (the values $\ell$ and $\vartheta$ may be not unique, but fixing one of them imposes a value on the another).

Theorem 3 Suppose Assumption 1 is satisfied, the variable $\Omega^{T} C^{T}$ is $P E$, i.e. there exist constants $\vartheta>0$ and $\ell>0$ such that

$$
\int_{t}^{t+\ell} \Omega(\tau)^{T} C^{T} C \Omega(\tau) d \tau \geq \vartheta I_{q} \quad \forall t \geq 0,
$$

and there exists a $n \times n$ matrix $W=W^{T}>0$ and constants $r>0, h>0$ such that

$$
\begin{gathered}
(A-L C)^{T} W+W(A-L C)+0.5 r \alpha^{2} C^{T} C \\
+h W B B^{T} W+\gamma \alpha^{-1} W \leq 0,
\end{gathered}
$$

where $\alpha=\gamma \eta^{-1} e^{2 \eta \ell}, \eta=-0.5 \ell^{-1} \ln \left(1-\frac{\gamma \vartheta}{1+\gamma^{2} \ell^{2}\|C \Omega\|^{4}}\right)$ and $\gamma>0$. Then in system (1), (2), (3)

$$
\begin{aligned}
{\left[\begin{array}{c}
|x(t)-z(t)| \\
|\tilde{\theta}(t)|
\end{array}\right] \leq } & (1+|| \Omega||)\left\{\sqrt{\frac{\alpha_{2}}{\alpha_{1}}} e^{0.5 \gamma \alpha^{-1} t}\left|\left[\begin{array}{c}
\delta(0) \\
\tilde{\theta}(0)
\end{array}\right]\right|\right. \\
& \left.+\sqrt{\frac{\alpha}{\alpha_{1} \gamma h}}\|v\|\right\}
\end{aligned}
$$

where $\alpha_{1}=\min \left\{\lambda_{\min }(W), 0.5 r \gamma^{-1}|| C \Omega \|^{-4}\right\}, \alpha_{2}=$ $\max \left\{\lambda_{\max }(W), 0.5 r\|C \Omega\|^{-2} \gamma^{-1} \alpha\right\}$.

PROOF. It follows from Assumption 1 that the variable $\Omega$ is bounded for any Hurwitz matrix $A-L C$ (the stability of this matrix follows from the Riccati equation for the matrix $W$ ), and it is also a continuous matrix function of time. Define $\rho=\|C \Omega\|<+\infty$. Consider as a candidate Lyapunov function $V(\delta)=\delta^{T} W \delta$, then

$\dot{V}=\delta^{T}\left[(A-L C)^{T} W+W(A-L C)\right] \delta+2 \delta^{T} W B v$.

Note that $2 \delta^{T} W B v \leq h \delta^{T} W B B^{T} W \delta+h^{-1} v^{T} v$, and therefore

$\dot{V} \leq \delta^{T}\left[(A-L C)^{T} W+W(A-L C)+h W B B^{T} W\right] \delta+h^{-1}|v|^{2}$.

Define $U(t, \delta, \tilde{\theta})=V(\delta)+r \rho^{-2} S(t, \tilde{\theta})$, then using the result of Lemma 1 we have

$$
\begin{aligned}
\dot{U} \leq & \delta^{T}\left[(A-L C)^{T} W+W(A-L C)+h W B B^{T} W\right] \delta \\
& -\gamma r \rho^{-2} \alpha^{-1} S+0.5 r \alpha^{2}|C \delta|^{2}+h^{-1}|v|^{2} \\
\leq & \delta^{T}\left[(A-L C)^{T} W+W(A-L C)+0.5 r \alpha^{2} C^{T} C\right. \\
& \left.+h W B B^{T} W\right] \delta-\gamma r \rho^{-2} \alpha^{-1} S+h^{-1}|v|^{2} \\
\leq & \delta^{T}\left[(A-L C)^{T} W+W(A-L C)+0.5 r \alpha^{2} C^{T} C\right. \\
& \left.+h W B B^{T} W+\gamma \alpha^{-1} W\right] \delta-\gamma \alpha^{-1} U+h^{-1}|v|^{2} \\
\leq & -\gamma \alpha^{-1} U+h^{-1}|v|^{2} .
\end{aligned}
$$

Note that according to the result of Lemma 1 the following inequalities hold:

$$
\begin{aligned}
& \lambda_{\min }(W)|\delta|^{2}+0.5 r \gamma^{-1} \rho^{-4}|\tilde{\theta}|^{2} \leq U(t, \delta, \tilde{\theta}) \\
& \leq \lambda_{\max }(W)|\delta|^{2}+0.5 r \rho^{-2} \gamma^{-1} \alpha|\tilde{\theta}|^{2} .
\end{aligned}
$$

Therefore, for all $\delta(0) \in \mathbb{R}^{n}$ and $\tilde{\theta}(0) \in \mathbb{R}^{q}$, and for all $t \geq 0$ we obtain:

$$
\left|\left[\begin{array}{c}
\delta(t) \\
\tilde{\theta}(t)
\end{array}\right]\right| \leq \sqrt{\frac{\alpha_{2}}{\alpha_{1}}} e^{0.5 \gamma \alpha^{-1} t}\left|\left[\begin{array}{c}
\delta(0) \\
\tilde{\theta}(0)
\end{array}\right]\right|+\sqrt{\frac{\alpha}{\alpha_{1} \gamma h}}\|v\|,
$$

and in addition

$|x(t)-z(t)| \leq|\delta(t)|+|| \Omega|||\tilde{\theta}(t)| \leq(1+|| \Omega||)\left|\left[\begin{array}{c}\delta(t) \\ \tilde{\theta}(t)\end{array}\right]\right|$,

which gives the required estimate.

This theorem estimates an upper bound on the $H_{\infty}$ gain of the observer from the input $v$ to the estimation errors $\tilde{\theta}$ and $x-z$. Only a minor modification of the LMI (6) in Theorem 3 is needed in order to ensure the $H_{\infty}$ gain from $v$ to a generic output $Z(x-z)$, where $Z$ is any matrix (this modification is omitted). Next, a minimization of the $H_{\infty}$ gain can be performed, using nonlinear optimization routines. 


\section{Sliding-mode adaptive observer}

In this case the observer in equation (2) is modified as follows:

$$
\begin{gathered}
\dot{z}=A z+\phi(y, u)+G(y, u) \hat{\theta}+L(y-C z)+\Omega \dot{\hat{\theta}} \\
+k B \operatorname{sign}[F(y-C z)],
\end{gathered}
$$

where $k>0$ is a design constant, $F \in \mathbb{R}^{s \times p}$ is a matrix to be designed and $\operatorname{sign}[v]=v /|v|$ for a vector $v \in \mathbb{R}^{m}$. Since (7) is discontinuous, solutions of this system are understood in a Filippov sense [11].

Theorem 4 Suppose assumption 1 is satisfied and let $k=\|v\|$. Assume the variable $\Omega^{T} C^{T}$ is PE, i.e. there exist $\vartheta>0$ and $\ell>0$ such that

$$
\int_{t}^{t+\ell} \Omega(\tau)^{T} C^{T} C \Omega(\tau) d \tau \geq \vartheta I_{q} \quad \forall t \geq 0
$$

and suppose there exists a matrix $W=W^{T}>0$, a matrix $F \in \mathbb{R}^{s \times p}$ and constants $r>0, \omega>0$ such that

$$
\begin{gathered}
(A-L C)^{T} W+W(A-L C)+\gamma \alpha^{-1} W \\
+\left(0.5 r \alpha^{2}+\omega\right) C^{T} C \leq 0, \\
W B=C^{T} F^{T}
\end{gathered}
$$

where

$$
\alpha=\gamma \eta^{-1} e^{2 \eta \ell}, \gamma>0, \eta=-0.5 \ell^{-1} \ln \left(1-\frac{\gamma \vartheta}{1+\gamma^{2} \ell^{2}\|C \Omega\|^{4}}\right) .
$$

Then in the system (1), (3), (7)

$$
\begin{aligned}
{\left[\begin{array}{c}
|x(t)-z(t)| \\
|\tilde{\theta}(t)|
\end{array}\right] \leq } & (1+|| \Omega||)\left(\sqrt{\frac{\alpha_{2}}{\alpha_{1}}} e^{-0.5 \gamma \alpha^{-1} \kappa t}\left|\left[\begin{array}{c}
\delta(0) \\
\tilde{\theta}(0)
\end{array}\right]\right|\right. \\
& \left.+2 \sqrt{\frac{\alpha}{\alpha_{1} \omega \gamma \kappa}}|F||| v \|\right),
\end{aligned}
$$

where $\kappa=\frac{0.5 r}{0.5 r+\omega\|C \Omega\|^{2}}$,

$$
\begin{gathered}
\alpha_{1}=\min \left\{\lambda_{\min }(W), \gamma^{-1}\left(0.5 r\|C \Omega\|^{-4}+\omega\right)\right\}, \\
\alpha_{2}=\max \left\{\lambda_{\max }(W), \gamma^{-1}\left(0.5 r\|C \Omega\|^{-2}+\omega\right) \alpha\right\} .
\end{gathered}
$$

PROOF. As in the previous theorem the variable $\Omega$ is bounded for any Hurwitz matrix $A-L C$ (the stability of this matrix follows from the Lyapunov equation for the matrix $W$ ) and let $\rho=\|C \Omega\|$. The error $\delta=x-z+\Omega \tilde{\theta}$ for the system (1), (3), (7) has the following dynamics:

$$
\dot{\delta}=(A-L C) \delta+B\{v-k \operatorname{sign}[F(y-C z)]\} .
$$

Consider three Lyapunov function components given by $V(\delta)=\delta^{T} W \delta, Z(\tilde{\theta})=\gamma^{-1} \tilde{\theta}^{T} \tilde{\theta}$ and $S(t, \tilde{\theta})=\tilde{\theta}^{T} S(t) \tilde{\theta}$. These functions have the property that

$$
\begin{aligned}
\dot{V}= & \delta^{T}\left[(A-L C)^{T} W+W(A-L C)\right] \delta+ \\
& +2 \delta^{T} C^{T} F^{T}\{v-k \operatorname{sign}[F(y-C z)]\} \\
\leq & -\delta^{T}\left[\gamma \alpha^{-1} W+\left(0.5 r \alpha^{2}+\omega\right) C^{T} C\right] \delta \\
& +2[y-C z]^{T} F^{T}\{v-k \operatorname{sign}[F(y-C z)]\} \\
& +2 \tilde{\theta}^{T} \Omega^{T} C^{T} F^{T}\{v-k \operatorname{sign}[F(y-C z)]\} \\
\leq & -\delta^{T}\left[\gamma \alpha^{-1} W+\left(0.5 r \alpha^{2}+\omega\right) C^{T} C\right] \delta \\
& +4\left|\tilde{\theta}^{T} \Omega^{T} C^{T}\right||F||| v|| \\
\dot{Z}= & 2 \tilde{\theta}^{T} \Omega^{T} C^{T}(C \delta-C \Omega \tilde{\theta}) \\
\leq & |C \delta|^{2}-\left|\tilde{\theta}^{T} \Omega^{T} C^{T}\right|^{2} \\
\dot{S} \leq & -0.5 \tilde{\theta}^{T} \tilde{\theta}+0.5 \rho^{2} \alpha^{2}|C \delta|^{2} .
\end{aligned}
$$

Introduce a new candidate Lyapunov function given by $U(t, \delta, \tilde{\theta})=V(\delta)+\omega Z(\tilde{\theta})+r \rho^{-2} S(t, \tilde{\theta})$, then we obtain

$$
\begin{aligned}
\dot{U} \leq & -\gamma \alpha^{-1} \delta^{T} W \delta-0.5 r \rho^{-2} \tilde{\theta}^{T} \tilde{\theta} \\
& +4\left|\tilde{\theta}^{T} \Omega^{T} C^{T}\right||F|\|v\|-\omega\left|\tilde{\theta}^{T} \Omega^{T} C^{T}\right|^{2} \\
\leq & -\gamma \alpha^{-1} \delta^{T} W \delta-0.5 r \rho^{-2} \tilde{\theta}^{T} \tilde{\theta}+4 \omega^{-1}|F|^{2}\|v\|^{2},
\end{aligned}
$$

where the inequality $4 s|F|\left|v\left\|-\omega s^{2} \leq 4|F|^{2}\right\| v \|^{2} / \omega\right.$, which is satisfied for all $s \in \mathbb{R}_{+}$, has been used in the last step of transformations. Since

$$
\begin{aligned}
\gamma^{-1}\left(0.5 r \rho^{-4}+\omega\right)|\tilde{\theta}|^{2} & \leq \omega Z(\tilde{\theta})+r \rho^{-2} S(t, \tilde{\theta}) \\
& \leq \gamma^{-1}\left(0.5 r \rho^{-2}+\omega\right) \alpha|\tilde{\theta}|^{2}
\end{aligned}
$$

we finally obtain

$$
\dot{U} \leq-\gamma \alpha^{-1} \kappa U+4 \omega^{-1}|F|^{2}\|v\|^{2},
$$

and for all $t \geq 0$

$\left|\left[\begin{array}{c}\delta(t) \\ \tilde{\theta}(t)\end{array}\right]\right| \leq \sqrt{\frac{\alpha_{2}}{\alpha_{1}}} e^{-0.5 \gamma \alpha^{-1} \kappa t}\left|\left[\begin{array}{c}\delta(0) \\ \tilde{\theta}(0)\end{array}\right]\right|+2 \sqrt{\frac{\alpha}{\alpha_{1} \omega \gamma \kappa}}|F|\|v\|$,

and again

$|x(t)-z(t)| \leq(1+\| \Omega||)\left|\left[\begin{array}{c}\delta(t) \\ \tilde{\theta}(t)\end{array}\right]\right|$

which gives the required estimate.

Remark 5 Compared to the case of Theorem 3, in Theorem 4, the accuracy of estimation is improved, since in part, the disturbance $v$ is compensated by $\operatorname{sign}(\cdot)$. Indeed, the dynamics of the state estimation error $e=x-z$ yields the following differential equation

$$
\begin{aligned}
\dot{e}= & \left(A-\left[L+\gamma \Omega \Omega^{T} C^{T}\right] C\right) e-G(y, u) \tilde{\theta} \\
& +B[v-k \operatorname{sign}(F C e)]
\end{aligned}
$$


which for $k=0$ reduces to (2). Consider the Lyapunov function $V(e)=e^{T} W e$, then

$$
\begin{aligned}
\dot{V} \leq & e^{T}\left\{(A-L C)^{T} W+W(A-L C)\right\} e \\
& +2 e^{T} C^{T} F^{T}(v-k \operatorname{sign}(F C e)) \\
& -2 e^{T} W\left[\gamma \Omega \Omega^{T} C^{T} C e+G(y, u) \tilde{\theta}\right] \\
\leq & e^{T}\left\{(A-L C)^{T} W+W(A-L C)\right\} e \\
& -2|F C e|(k-\|v\|) \\
& -2 e^{T} W\left[\gamma \Omega \Omega^{T} C^{T} C e+G(y, u) \tilde{\theta}\right] .
\end{aligned}
$$

Therefore, for the observer in (7), with $k=\|v\|$, the term proportional to $|F C e|$ disappears and the influence of $v$ is canceled. The Lyapunov equation for the matrix $W$ is also simpler than in the case of Theorem 4 (and an additional decision variable $F$ appears, offering more flexibility to tune the algorithm).

Remark 6 The introduction of the "structural constraint" $W B=C^{T} F^{T}$ in (8) imposes a limitation on the class of triple $(A, B, C)$ to which these results are applicable (specifically minimum phase and relative degree one conditions for the system (1) with respect to the output $y$ and the input $v$ [6], or with the hyper minimum phase property [7]). A constructive approach to solving such problems is given in [6]. Equivalent conditions can be found in Lemma 1 of [7]. If these conditions are not met, then "approximate" versions can be employed which reject "most" of the disturbance [2].

Remark 7 If the state $x(t)$ is bounded, i.e. $\|x\|<+\infty$, then it is possible prove boundedness of all variables in the system (without such an assumption the variable $z(t)$ following $x(t)$ may be unbounded).

\section{Conclusions}

The problem studied in this paper is that of improving the performance of the adaptive observer originally proposed in [20]. Following [18], a scheme augmented by including a sliding mode term is proposed. First, the $H_{\infty}$ gain with respect to external disturbances is evaluated to minimize the effect of disturbances on the output errors in a $H_{\infty}$ sense. Next, a sliding-mode modification of the adaptive observer equations from [20] is proposed in order to improve disturbance attenuation. The conditions for stability of the new scheme have been established.

\section{References}

[1] A. Alessandri and A. Rossi. Time-varying increasing-gain observers for nonlinear systems. Automatica, 49(9):28452852,2013

[2] H. Alwi, C. Edwards, and C.P. Tan. Sliding mode estimation schemes for incipient sensor faults. Automatica, 45(7):1679$1685,2009$.
[3] B.D.O. Anderson. Exponential stability of linear equations arising in adaptive identification. IEEE Trans. Automat. Control, 22:83-88, 1977.

[4] G. Besanon, editor. Nonlinear observers and applications, volume 363 of Lecture Notes in Control and Inforamtion Science. Springer Verlag, Berlin, 2007.

[5] N. Boizot, E. Busvelle, and J.-P. Gauthier. An adaptive highgain observer for nonlinear systems. Automatica, 46(9):14831488, 2010.

[6] C. Edwards, Xing-Gang Yan, and S.K. Spurgeon. On the solvability of the constrained Lyapunov problem. Automatic Control, IEEE Transactions on, 52(10):1982-1987, 2007.

[7] D. Efimov and A. Fradkov. Adaptive tuning to bifurcation for time-varying nonlinear systems. Automatica, 42:417-425, 2006.

[8] D. Efimov and A.L. Fradkov. Design of impulsive adaptive observers for improvement of persistency of excitation. International Journal of Adaptive Control and Signal Processing, 29(6):765-782, 2015.

[9] D.V. Efimov. Robust adaptive nonlinear partial observers for time-varying chaotic systems. In Proc. IEEE CDC, Atlantis, Paradise Island, Bahamas, December 2004. IEEE.

[10] M. Farza, I. Bouraoui, T. Ménard, R. Ben Abdennour, and M. M'Saad. Adaptive observers for a class of uniformly observable systems with nonlinear parametrization and sampled outputs. Automatica, 50(11):2951-2960, 2014.

[11] A.F. Filippov. Differential Equations with Discontinuous Right-hand Sides. Kluwer Academic Publishers, 1988.

[12] A.L. Fradkov, H. Nijmeijer, and A.Yu. Markov. Adaptive observer-based synchronization for communications. Int. J. Bifurcat. Chaos, 10(12):2807-2814, 2000.

[13] A.L. Fradkov, V.O. Nikiforov, and B.R. Andrievsky. Adaptive observers for nonlinear nonpassifiable systems with application to signal transmission. In Proc. 41th IEEE Conf. Decision and Control, pages 4706-4711, Las Vegas, Dec. 2002. IEEE.

[14] P. Ioannou and J. Sun. Robust Adaptive Control. Prentice Hall, Inc., 1996.

[15] R.G. Sanfelice and L. Praly. On the performance of high-gain observers with gain adaptation under measurement noise. Automatica, 47(10):2165-2176, 2011.

[16] Y. Shtessel, C. Edwards, and L. Fridman. Sliding Mode Control and Observation. Birkhauser, 2013.

[17] A. Xu and Q. Zhang. Residual generation for fault diagnosis in linear time-varying systems. IEEE Trans. Autom. Control, 49(5):767-772, 2004.

[18] X.G. Yan and C. Edwards. Fault estimation for single output nonlinear systems using an adaptive sliding mode observer. IET Control Theory and Applications, 2:841-850, 2008.

[19] J. S.-C. Yuan and W.M. Wonham. Probing signals for model reference identification. IEEE Trans. Automat. Control, 22:530-538, 1977.

[20] Q. Zhang. Adaptive observer for multiple-input-multipleoutput (MIMO) linear time varying systems. IEEE Trans. Autom. Control, 47(3):525-529, 2002. 